\title{
The role of boundary conditions on the dynamics of green coffee beans in a rotated dryer
}

\author{
Machado, M.V.C.; Resende, I.A.; Lima, R.M.; Brandão, R.J.; Pivello, M.R.; Nascimento, \\ S.M.; Duarte, C.R., Barrozo, M.A.S. ${ }^{*}$ \\ ${ }^{a}$ Federal University of Uberlândia, Chemical Engineering School, Uberlândia/MG, Brazil. \\ *E-mail of the corresponding author: masbarrozo@ufu.br
}

\begin{abstract}
Coffee drying and roasting are usually performed in rotated dryers; therefore, the study of particle dynamics in this equipment is of great relevance to improve their efficiency and hence the quality of the final product. Thus, this work aimed to investigate experimentally and numerically the dynamics of coffee beans in a rotary dryer. The Euler-Euler model was employed to reproduce the particle velocity profile in the rolling regime under different boundary conditions. The results shown that the lower specularity coefficient (0.01), which characterizes the smooth wall and free slip condition, reproduced the bed behavior that most resembled the experimental one. On the other hand, the other coefficients (0.1 and 1.0) showed an increasing deformation in the bed surface, different from the observed experimental behavior. It was also verified that, as the filling degree increases, the bed surface deformation becomes more pronounced.
\end{abstract}

Keywords: coffee; rotated dryer; simulation; specularity coefficient. 


\section{Introduction}

During the processing of coffee beans, roasting and drying can be performed in rotary drums. These equipment also have a wide range of applications in the industry, such as mixing, drying, granulation, milling and coating. Rotary drums can process many types of raw materials, with large size distributions and different physical properties.

The efficiency of rotatory drums, depending on the process in question, is highly dependent on the granular movement in the interior, which in turn is related to the energy, mass and momentum transfer rates ${ }^{[1]}$. Therefore, to improve the efficiency of these equipment requires detailed information about the movement of the particles inside.

Rotating drums can show seven different flow regimes (sliding, surging, slumping, rolling, cascading, cataracting and centrifuging), which depend on the rotational speed, filling degree, physical properties of granular materials and drum geometry ${ }^{[2]}$. Each one with its own specific flow behavior, which increase the complexity in its study.

Cristo et al. ${ }^{[3]}$ recommend the rolling regime for coffee roasting, in order to ensure a uniform heat transfer within the equipment. This regime, which is studied in the present work, is characterized by two different regions: a passive region, found near the drum wall, where particles move as a solid body, and an active region, found near the bed material surface, where the particles avalanche and cascade downward. The physical mechanisms such as, mixing and segregation, heat and mass transfer, and so on, mainly occur in the active region.

Parallel to experimental studies, the numerical simulations arise as a complementary tool in the granular flows investigation. In the present paper, the granular flow was simulated using the EulerEuler approach along with the kinetic theory of granular flow. In this kind of simulation both phases are treated as interpenetrating continua and the Eulerian approach solves a set of moment and continuity equations for each phase. However, the simulated results are very sensitive to the selected boundary conditions. So, the proper choice of these boundary condition on the wall is of crucial importance to represent the experimental behavior.

In the context of the multiphase granular model, the specularity coefficient appears as one of the boundary conditions to be used. The specularity coefficient is a representation of the fraction of collisions between the particle and wall (contour), which transfer lateral momentum to the wall ${ }^{[4]}$. This coefficient is closely related to the flow conditions and the properties of the wall and there are no experimental determination about it reported in the literature. The value of the specularity coefficient $(\varphi)$ depends on the sliding condition of the wall and its roughness. Thus, it can range from 0 , for perfectly specular collisions, with a smooth wall condition and free slip, and 1, for perfectly diffuse collisions with rough wall and zero slip ${ }^{[5]}$.

Despite the relevance of the boundary conditions for Eulerian simulations, there are few studies about the influence of interactions between particles and the wall on the behavior of the solid phase in rotary dryers. Therefore, the present paper aims to investigate the effect of the boundary conditions on the simulation results of the movement of coffee particles during the rolling regime in a rotating dryer, comparing the simulation with the experimental behavior. 


\section{Materials and Methods}

\subsection{Experimental Setup}

Green coffee beans were the particulate material used in this study, with the following specifications: average diameter $5.24 \mathrm{~mm}$, density $1368.3 \mathrm{~kg} / \mathrm{m}^{3}$, sphericity 0.88 , moisture (dry base) equal to 12.15 and angle of repose of $38.4^{\circ}$. The experimental tests were conducted in a stainless steel rotating dryer, with the following dimensions: diameter $21.5 \mathrm{~cm}$ and length $50 \mathrm{~cm}$. The front and back walls of the cylinder are made of transparent glass that allows the visualization of the coffee beans movement inside the drum. The inner wall of the dryer was coated with a P80 sandpaper.

The particle velocity profiles were obtained by the image analysis technique using a high speed camera (up to 2000 frames/s). The videos were recorded for the following filling degrees:10, 20, 30,40 and $50 \%$ and at a rotational speed of $12.5 \mathrm{rpm}$, which characterizes the rolling regime.

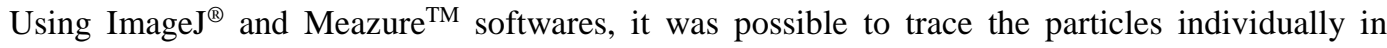
different picture frames. Thus, the velocity of each particle was determined by subtracting the positions of the same particle in two distinct frames. That is, immediately after x2 and y2 (see Figure 1); and before $\mathrm{x} 1$ and $\mathrm{y} 1$ of the reference line, and then dividing this result by the time interval between the respective frames (t2-t1). Figure 1 shows the positioning of the reference line over the bed radius (r), along which the velocity were measured. From these profiles, it is possible to find the location of the interface between the active and passive layers along the reference line, determined by the point of inversion of the direction of displacement of the particles. In other words, the point where the particles velocity is nearly zero and delimits the transition between the two regions of rolling regime (active and passive) ${ }^{[8]}$.

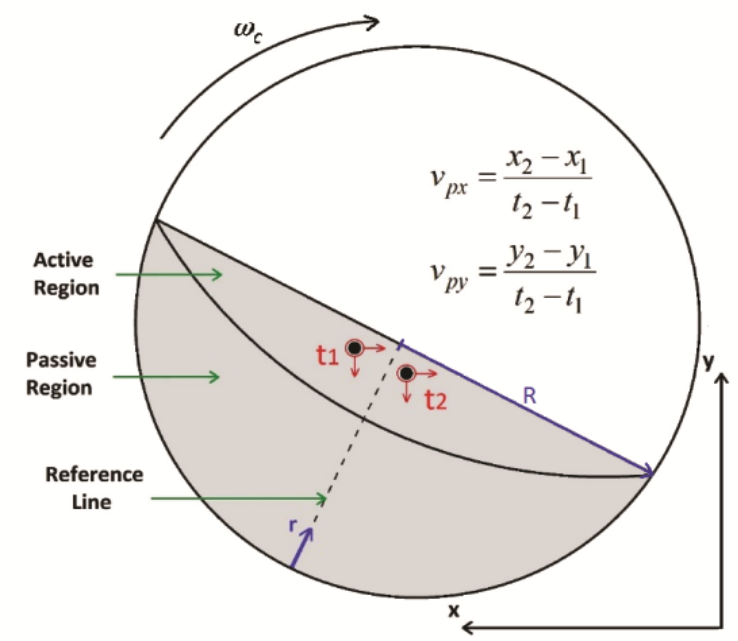

Fig. 1: Methodology for particle velocity measurement during the rolling regime. 


\subsection{Numerical Methodology}

All simulations were performed in Fluent ${ }^{\circledR} 14.0$ software, using the Eulerian methodology and the Eulerian Granular Multiphase Model to characterize the fluid and the granular phase behavior inside the rotating dryer. To describe the interactions between the solid phases (particle-particle and particle-wall), it was used the Granular Kinetic Theory. For the granular viscosity, the model of Syamlal et al. ${ }^{[6]}$ was used. For the bulk granular viscosity, the solids pressure and the radial distribution function, the models of Lun et al. ${ }^{[7]}$ were used.

The no-slip condition was used to describe the boundary conditions in the wall relative to the fluid phase. Meanwhile, for the solid phase, it was used the boundary conditions given by the specularity coefficient ${ }^{[5]}$. Equation 1 expresses the tangential velocity of the solid phase at the wall $\left(u_{s w}\right)$ in terms of specularity coefficient $(\varphi)$.

$$
u_{s w}=\frac{-6 \alpha_{S, \max } \mu_{S}}{\sqrt{3} \sqrt{\theta_{S}} \pi \varphi \rho_{S} \alpha_{S} g_{0, s s}} \frac{\partial u_{s w}}{\partial n}
$$

Where $\alpha_{S}$ and $\rho_{S}$ are the volume fraction and the solid phase density, respectively. $\theta_{S}$ and $\mu_{S}$ are the granular temperature and solid viscosity, $g_{0, S s}$ is the radial distribution function.

\section{Results and Discussion}

\subsection{Analysis of the wall boundary conditions: specularity coefficient}

For the purpose to verify the effect of the wall boundary conditions, different values of specularity coefficient $(\varphi)$ were analyzed in the Eulerian simulation of the coffee beans in a rotary dryer. Hereafter, it is presented a qualitative analysis of the experimental and numerical results, the latter being obtained for the specularity coefficients equal to 1.0; 0.1 and 0.01 . Figures 3, 4, 5, 6 and 7 show the distribution of the solid phase in the front wall of the dryer and also in the transverse plane located in half the length, for filling degrees of 10, 20, 30, 40 and $50 \%$, respectively.

From the Figures 2, 3, 4, 5 and 6, it is possible to note that the simultions with the lowest specularity coefficient value, which characterizes the smooth wall and free-slip condition, better reproduced the experimental behavior, for all filling degrees values evaluated. Concerning to the other specularity coefficients values (0.1 and 1.0), it also can be observe an increasing in bed surface deformation, presenting a divergence from the observed experimental behavior. This is due to the fact that the highest specularity coefficient (1.0) characterizes the rough wall condition, resulting in a high friction between the wall and the particles, and carrying the particles at higher heights. Also, it can be verified that as the filling degree increases, this bed surface deformation becomes more pronounced.

Comparing the solid fraction distributions in the front wall and in the plane at the half length of the dryer, it can be observed a difference in the bed behavior only for the specularity coefficient equal to 1.0. For low values of this parameter, this wall effect is not perceived, since the solid distribution in the wall and in the plane in the middle are practically coincident. 


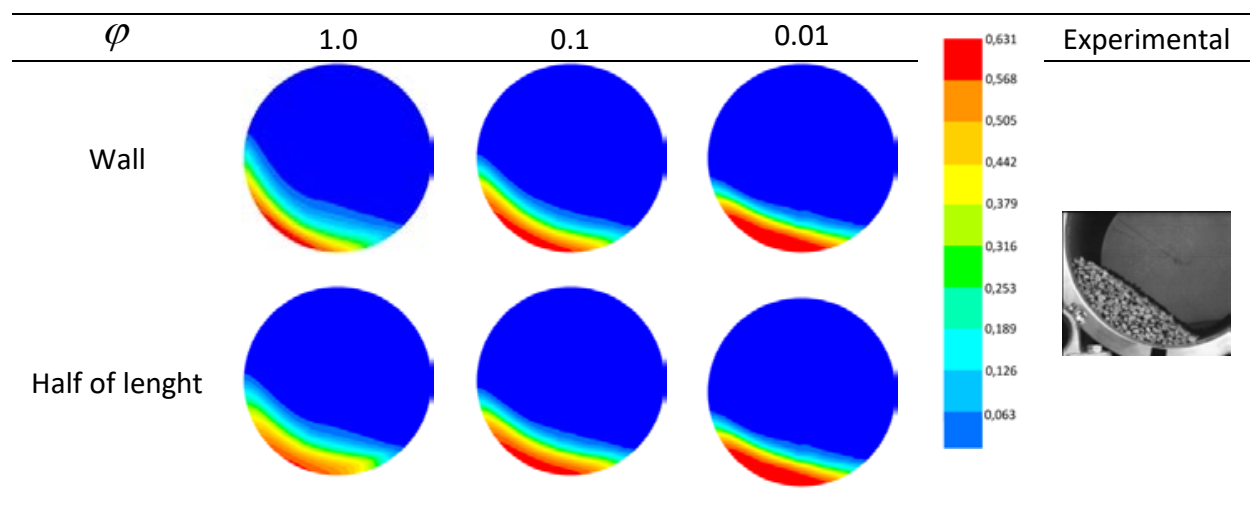

Fig. 2: Solids volume fraction for different values of specularity coefficient at $f=10 \%$

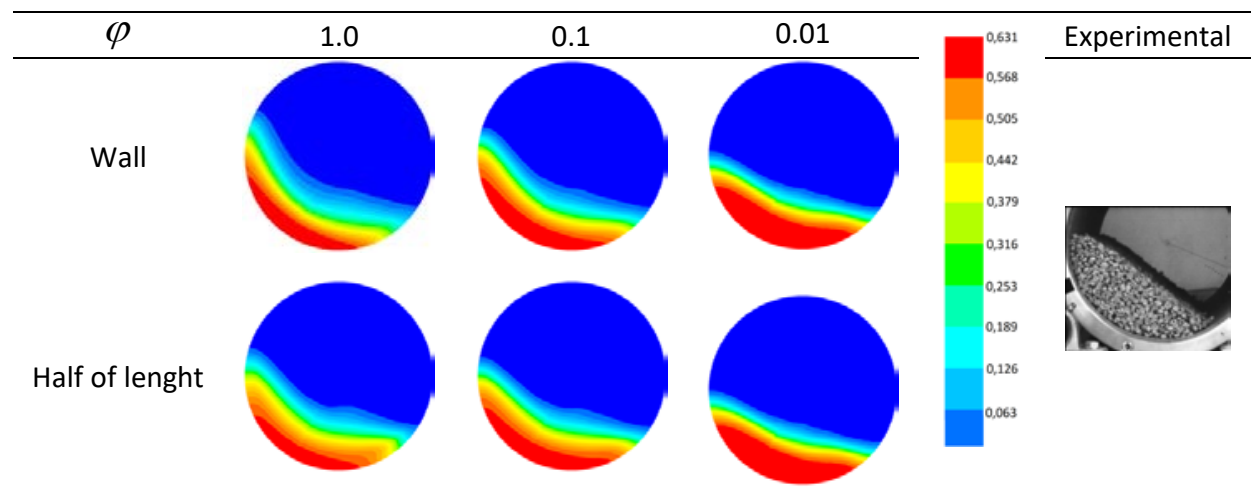

Fig. 3: Solids volume fraction for different values of specularity coefficient at $f=20 \%$

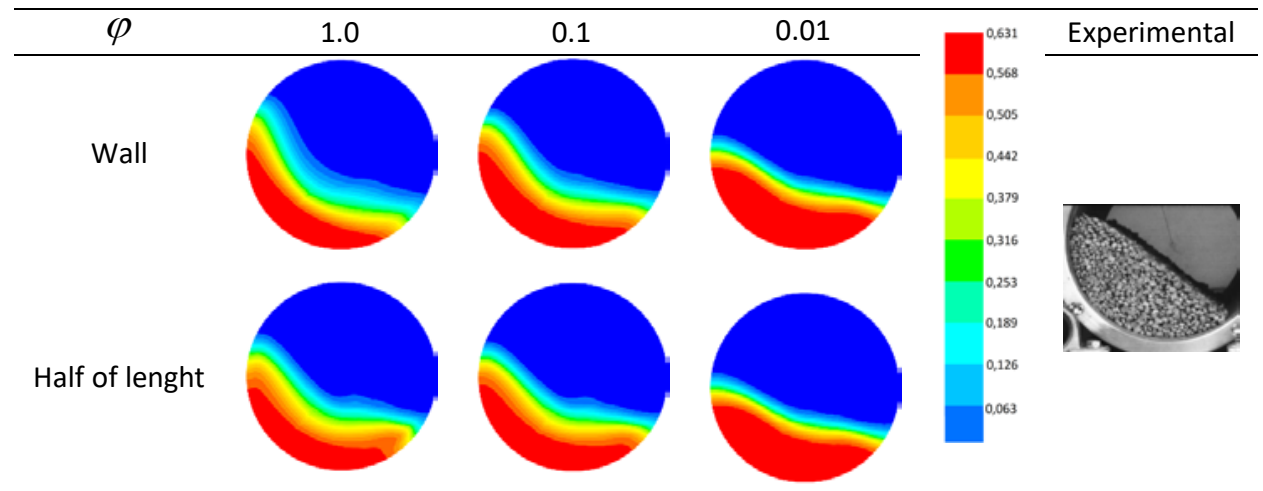

Fig. 4: Solids volume fraction for different values of specularity coefficient at $f=30 \%$ 


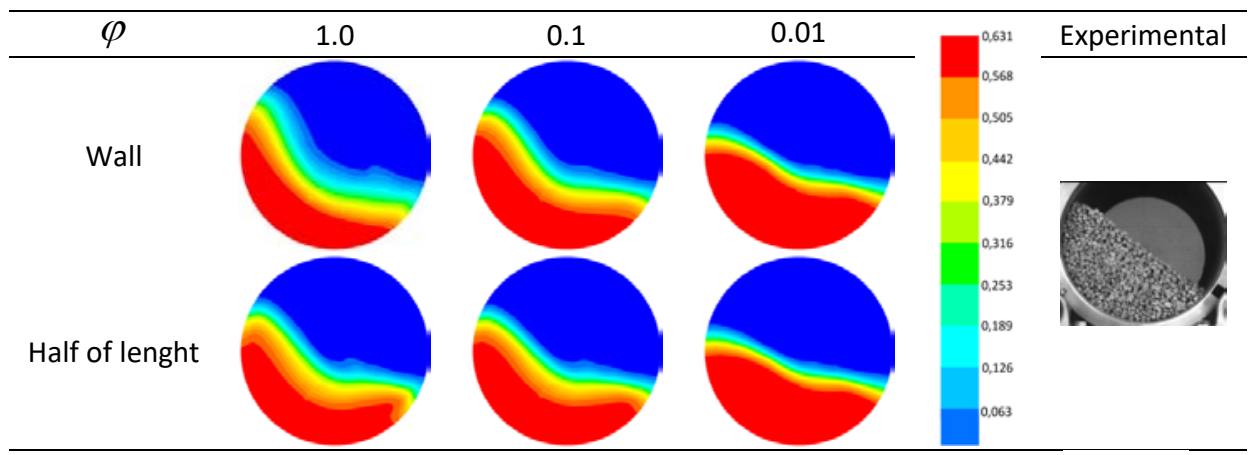

Fig. 5: Solids volume fraction for different values of specularity coefficient at $f=40 \%$

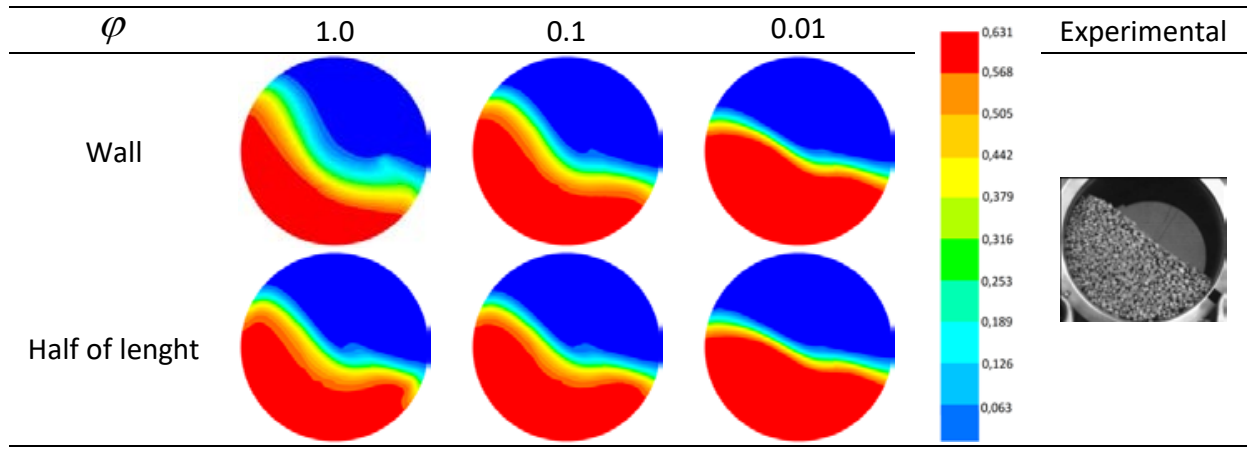

Fig. 6: Solids volume fraction for different values of specularity coefficient at $f=50 \%$

\subsection{Particle velocity profiles}

In addition to the qualitative analysis, it was also present a quantitative analysis of the particle velocity obtained by the simulation using different values of specularity coefficients. Figure 7 (a), (b), (c), (d) and (e) show the experimental and simulated results of the velocity profile of coffee beans, for filling degrees of 10, 20,30, 40 and 50\%, respectively.

Figure 7 results show that, for all filling degrees, the transition from the passive to the active layer obtained by simulation occurs at radial positions smaller than those obtained experimentally. Thus, the point of inversion of the direction of displacement of the particles is underestimated by the numerical model. This evidence is more pronounced for the lower specularity coefficient (0.01), which is justified by the greater slippage in the wall, characterized by this condition.

Moreover, it is noted that for the greater filling degrees, the behavior of the simulations performed with the higher specularity coefficients values ( 0.1 and 1.0$)$, becomes more similar in the passive region. Such similarity is also observed for the active region in all evaluated filling degree values. Although there is a large difference between these two specularity coefficients values, the results presented by them are quite similar, suggesting that simulations performed within this range of values $(0.1-1.0)$ would not result in different information. 
Machado, M.V.C., Resende, I.A., Lima, R.M., Brandão, R.J., Pivello, M.R., Nascimento, S.M., Duarte, C.R., Barrozo, M.A.S.
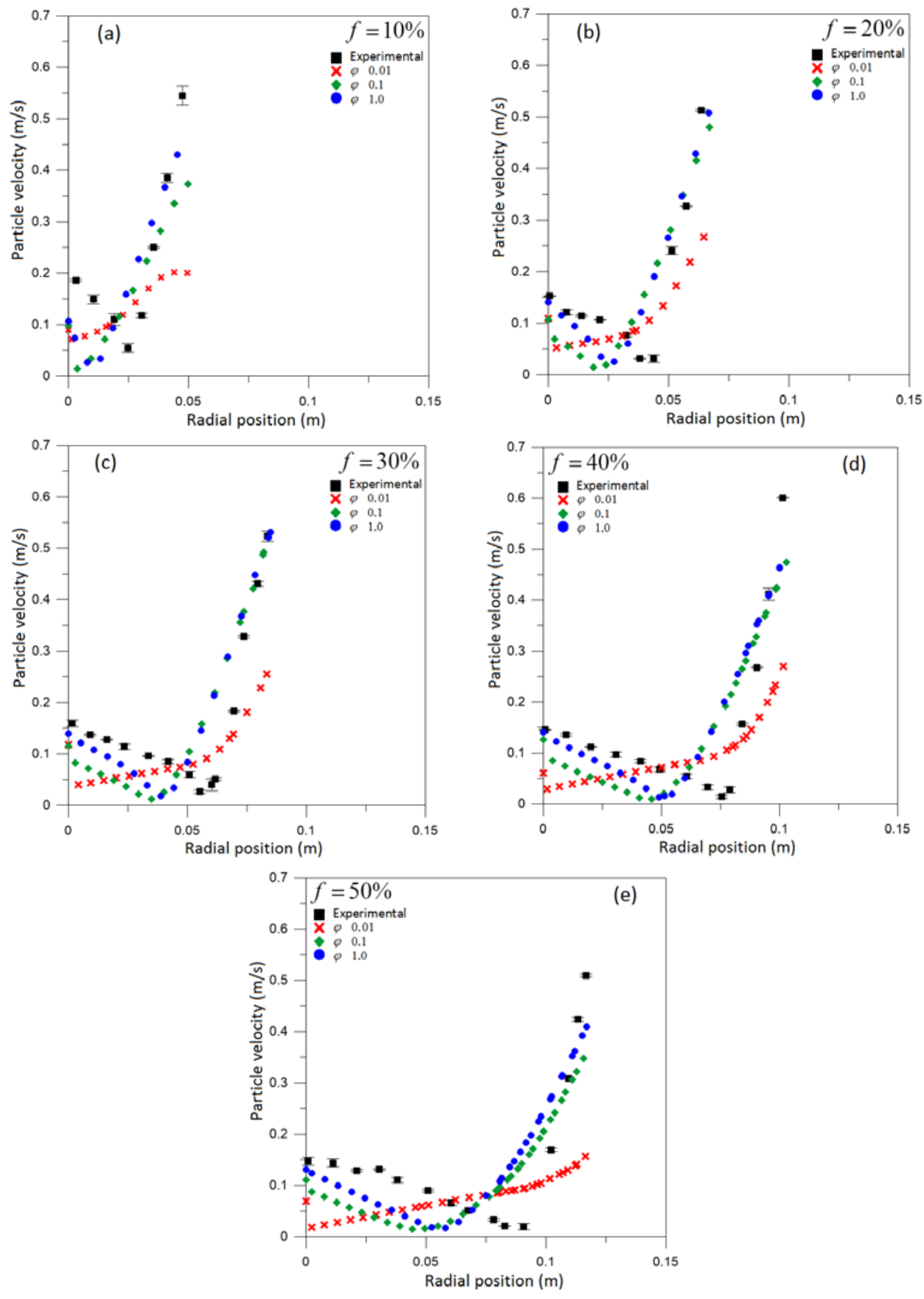

Fig. 7: Experimental and numerical particle velocity profiles for different filling degrees. 


\section{Conclusions}

This work assessed the boundary conditions effects on the coffee beans dynamics inside a rotating dryer by comparing numerical results with experimental data. It was observed that the lower specularity coefficient (0.01), which characterizes the smooth wall and free slip condition, reproduced the qualitative bed behavior that most resembled the experimental one. On the other hand, the other coefficients ( 0.1 and 1.0 ) showed an increasing deformation in the bed surface, different from the observed experimental behavior. It was also verified that, as the filling degree increases, the bed surface deformation becomes more pronounced. However, the particle velocities profiles obtained by simulation with this lower specularity coefficient lead to a transition from the passive to the active layer at radial positions smaller than those obtained experimentally.

\section{Acknowledgments}

The authors would like to thank FAPEMIG, CNPq and CAPES for the financial resources assigned to carry out this work.

\section{References}

[1] SANTOS, D.A., PETRI, I.J., DUARTE, C.R., BARROZO, M.A.S. Experimental and CFD study of the hydrodynamic behavior in a rotating drum. Powder Technology 2013, 250, 52-62.

[2] MELLMANN, J. The transverse motion of solids in rotating cylinders - forms of motion and transition behavior. Powder Technology. 2001, 118, 251-270.

[3] CRISTO, H.P., MARTINS, M.A., OLIVEIRA, L.S., FRANCA, A.S, Transverse flow of coffee beans in rotating roasters, Journal of Food Engineering 2006, 75, 142-148.

[4] LI, T.; BENYAHIA, S., Revisiting Johnson and Jackson Boundary Conditions for Granular Flows, American Insitute of Chemical Engineers (AIChE) 2011,58 (7), 2058-2068.

[5] JOHNSON, P.C., e JACKSON, R., Frictional-Colisional Constitutive Relations for Granular Materials with Application to Plane Shearing, J. Fluid Mech. 1987, 176, 67-93.

[6] SYAMLAL, M., ROGERS, W., O’BRIEN, T.J, MFIX documentation: Theory Guide, National Technical Information Service, vol. 1, U.S. Department of Energy, Springfiled, USA, 1983.

[7] LUN, C.K.K., SAVAGE, S.B., JEFFREY, D.J., CHEPURNIY, N. Kinetic theories for granular flow: inelastic particles in coquette flow and singly inelastic particles in a general flow fiel, J. Fluid Mech, 1984, 140, 233-256.

[8] MACHADO, M.V.C., NASCIMENTO, S.M., DUARTE, C.R., BARROZO, M.A.S., Boundary conditions effects on the particle dynamic flow in a rotary drum with a single flight, Powder Technology 2017, 317, 341-349. 